\title{
"Sometimes I Feel Grateful...": Experiences of the Adolescent Siblings of Children with Autism Spectrum Disorder in Malaysia
}

\author{
Shin Ying Chu ${ }^{1} \mathbb{D}$. Siti Nur Zafirah binti Kassim ${ }^{1}$. Chun Hong Gan ${ }^{2}$. Veronica Fierro ${ }^{3}$ Caryn Mei Hsien Chan $^{4}$. \\ Deborah Hersh ${ }^{5}$ (i)
}

Accepted: 30 June 2021 / Published online: 10 July 2021

(c) The Author(s), under exclusive licence to Springer Science+Business Media, LLC, part of Springer Nature 2021

\begin{abstract}
Sibling relationships remain understudied in typically developing adolescents who have siblings with autism spectrum disorder (ASD). This study explored the lived experiences and perceptions of adolescents who have a brother or a sister with a diagnosis of ASD using semi-structured interviews. Using thematic analysis, the participants reported having difficulties communicating with their siblings. Feeling a mixture of emotions was common when interacting with their siblings with ASD and emotional self-regulation was a typical coping strategy utilized by these adolescents. Despite the challenges, having a sibling with ASD led to closer relationships between the siblings. These findings offer insights into these adolescents' needs and how they may be best supported, informing the practice of speech-language pathology and other health professions.
\end{abstract}

Keywords Autism $\cdot$ Siblings $\cdot$ Communication $\cdot$ Challenges $\cdot$ Interview

\section{Introduction}

With the prevalence of ASD continuing to rise (Chiarotti \& Venerosi, 2020; Lord et al., 2020), greater numbers of families are being affected. Therefore, there is a growing need to evaluate the impact of associated difficulties of the diagnosis on the immediate family. Adolescent, typically developing (TD) siblings of individuals with ASD may experience significant stress and anxiety (Gorjy et al., 2017; Koukouriki

Shin Ying Chu

chushinying@ukm.edu.my

Siti Nur Zafirah binti Kassim

sitinur.zafirah97@gmail.com

Chun Hong Gan

ganchunhong@ukm.edu.my

Veronica Fierro

Veronica.Fierro@ rockhurst.edu

Caryn Mei Hsien Chan

caryn@ukm.edu.my

Deborah Hersh

d.hersh@ecu.edu.au

1 Faculty of Health Sciences, Centre for Healthy Ageing and Wellness (H-CARE), Speech Sciences Programme, Universiti Kebangsaan Malaysia, Jalan Raja Muda Abdul Aziz, 50300 Kuala Lumpur, Malaysia
\& Soulis, 2020). These TD siblings face unique challenges associated with having a sibling with ASD as opposed to other developmental disabilities (12-17 years old in Gorjy et al., 2017; 12-20 years old in Corsano et al. 2016). For example, they experience embarrassment in social situations and public places due to people's reactions towards the behavior of their siblings with ASD (Pavlopoulou \& Dimitrou, 2020; Leedham et al., 2020; Noonan et al., 2018). The challenges associated with the social-communication

2 Faculty of Health Sciences, Centre for Rehabilitation and Special Needs, Clinical Psychology and Behavioral Health Programme, Universiti Kebangsaan Malaysia, Jalan Raja Muda Abdul Aziz, 50300 Kuala Lumpur, Malaysia

3 Department of Communication Sciences and Disorders, Rockhurst University, 1100 Rockhurst Road, Kansas City, MO 64110, USA

4 Faculty of Health Sciences, Centre for Community Health Sciences (ReaCH), Health Education Programme, Universiti Kebangsaan Malaysia, Jalan Raja Muda Abdul Aziz, 50300 Kuala Lumpur, Malaysia

5 School of Medical and Health Sciences, Edith Cowan University, 270 Joondalup Drive, Joondalup, WA 6027, Australia 
impairment and problematic behavior of children with ASD may impact family members and family dynamics (Jones et al., 2019).

These challenges may impact these siblings' relationships, particularly in how they spend time together as they move into adulthood (Travers et al., 2020). Specifically, of 159 TD siblings between 18-30 years old living in Tennessee, United States of America [USA]), only 12 reported doing activities together with their sibling with ASD, including talking on the phone, taking art, music, or dance lessons, and other activities (e.g., going grocery shopping, playing sports) (Travers et al., 2020). The difficulties of social interaction between TD siblings and their sibling with ASD have also been considered in a number of studies focusing on children and adolescents. For example, social and interactional issues can contribute to poor psychological well-being in the TD siblings (4-18 years old in the study by Jones et al., 2019; participants as young as 6 years old in the study by Habelrih et al., 2018). Among the adolescent siblings, feelings of fear, anxiety, and social isolation have been reported by those between 11 and 16 years old from the United Kingdom and the USA when their sibling with ASD behaved inappropriately (Moss et al., 2019). Negative acts of others towards their sibling with ASD have also led to feelings of anger, frustration, and disappointment in 12- to 19-yearold TD siblings (Dongola, 2018). In addition, TD siblings between 7 to 18 years old often experience social communication difficulties due to difficulty with reciprocal conversations with their sibling with ASD (Ward, 2016). Recently, an Italian study of 44 TD siblings ( 25 females and 19 males, aged between 6 and 17 years old) of a sibling with ASD used both the Sibling Relationship Inventory (Stocker \& McHale, 1992) and analysis of drawings of themselves with their sibling with ASD (Guidotti et al., 2021). It showed that there was affection for the sibling with ASD, although male TD siblings demonstrated more conflict than the female TD siblings. Taken together, these study results suggest that there is a need for intervention to facilitate interactions between TD siblings and their siblings with ASD to enhance the building of a better sibling relationship.

According to Family Systems Theory, the family is a whole system and is affected by the relationships between family members (Laghi et al., 2017). That is, the relationship between one system, such as that between siblings, can impact the entire family system. Sibling relationships have a central role in family life (Shali \& Patil, 2017). A sibling is an early peer who provides important opportunities for social interaction given the amount of time that is shared between siblings (Johnson et al., 2020). As an earliest social partner, siblings play a significant role in influencing each other's lives as they experience growing up together and are regularly interacting with each other (Hall, \& Rossetti, 2018; McHale et al., 2016). They support each other to develop perspective-taking, conflict resolution, and communication skills (Hughes, McHarg, \& White, 2017). The siblings of individuals with special needs go through life events and personal experiences that are not usually experienced by their peers (Gan, 2017). In fact, for families who have children with ASD, the sibling relationship is not only the first relationship, but a powerful one as well (GordonPershey, \& Hodge, 2017). Due to the high needs and complex nature of ASD, 14- to 18-year-old TD siblings from the USA have reported receiving reduced parental attention, sometimes leading to a dislike towards their sibling with ASD (Henderson-Rudling, 2019). Nevertheless, others (from Australia) have reported experiencing supportive and warm relationships between siblings (Cridland et al., 2016). Note that these studies were conducted across the United Kingdom, the United States, and Australia (Petalas et al., 2012; Moss, 2018) and to date, studies on the challenges and impact of having a sibling with ASD have not been conducted in Malaysia.

In one study conducted in Malaysia, in which parents of children with ASD were interviewed, they discussed their lack of knowledge about ASD, the impact of having a child with ASD in their lives, their experience with multiple barriers in seeking professional advice, and the positive aspects of receiving speech-language therapy (Chu et al., 2020a, 2020b). Nevertheless, in addition to gaining insight from parents, it is essential to understand the experiences of siblings as well, who are also integral to family systems. Differences in experiences with regard to having a sibling with ASD have been documented between TD younger siblings and TD older siblings. For instance, in terms of the coping strategies used, younger siblings (9-10 years old) often expressed feelings of anger and upset, whereas older siblings (11-17 years old) adapted avoidant strategies to avoid conflict (Tsai Cebula et al. 2018). Younger siblings (2-5 years younger than their sibling with ASD) also expressed a more self-centered wish regarding the decision to put their sibling with ASD in a group home, while older siblings (1-19 years older than their sibling with ASD) took into consideration their parents' situation (Benderix, \& Sivberg, 2007). While middle childhood siblings (8-12 years of age) reported a need to increase their knowledge about their sibling's disability (Petalas et al., 2009), adolescent siblings (14-17 years old), did not report such a need (Petalas et al., 2012). This suggests that challenges faced at different developmental stages will have different needs, which parents and professionals should be aware of during intervention.

To ensure the effectiveness of speech-language pathology (SLP) service delivery, therapists and families need to work together to understand the individual child's condition better. To maximally support this collaboration, therapists must take the initiative to educate themselves about the challenges and attitudes of families so that needs and concerns are met. 
This includes considering the perspectives of TD siblings of children with ASD who spend time playing and interacting with their sibling with ASD at home and growing older together. In Asian culture, it is common for siblings to take care of each other (especially caring for those with disabilities or disease) when parents pass away (Wu et al., 2018). Often, this is the responsibility of the elder son or sibling in the family (Kale \& Aslan, 2020). Like most countries in Asia, in Malaysia, high expectations are placed on elder siblings to care for their younger siblings in a family system (Nuckolls, 1993). For example, they are expected to assist their parents with housework and looking after their younger siblings. As sibling relationships are shaped by their macrosystem-level cultural contexts (Cicirelli, 1994), knowing the needs of adolescent TD siblings with a sibling with ASD at an early stage could assist in developing a more comprehensive support system for the TD siblings. In Malaysia, limited studies have been conducted to determine the challenges or issues faced by families of children with ASD, and although studies have been conducted in Western countries, few were focused on adolescent TD siblings. There is a need to examine this topic within the Malaysian context given its collectivist culture, beliefs and values attributable, in part, to a heterogenous population (in terms of race and religion), which likely strongly influences the family as a social unit (Sumari et al., 2019). Understanding the challenges faced by adolescent TD siblings would help healthcare professionals, particularly speech-language therapists, occupational therapists, and physical therapists to target these issues to improve service delivery.

Based on the Family Systems Theory, which focuses on the examination of relationships within the family, such as maternal, marital, and/or sibling subsystems, this qualitative study aimed to explore specifically the experiences and perceptions of TD adolescents with siblings with ASD. Given that the Family Systems Theory emphasizes all the systems in the family interact to influence each other's, practitioners will be more effective when viewing the family as a whole system rather than focusing only on parent-child interactions. Qualitative research is recognized for obtaining valuable information and capturing in-depth knowledge when exploring subjective experiences (Willig \& Stainton-Rogers, 2008). An in-depth exploration using semi-structured interviews to obtain the perspectives of these adolescent TD siblings can deepen our understanding of the difficulties and issues they face, allowing allied health professionals to be better able to address these areas for improvement. Our goal is to understand the challenges, coping strategies, and impact on relationships for adolescent TD siblings growing up with siblings with ASD in a Malaysian context. Considering that communication and social challenges are key features in children with ASD, this study seeks to understand how these aspects impact on their adolescent TD siblings in order to assist the development of a targeted intervention for both the children with ASD and their siblings, thereby promoting new avenues for support.

\section{Methods}

\section{Participants}

Fourteen adolescent siblings (mean age $=15.4, \mathrm{SD}=2.06$, 11 females) of individuals with ASD from nine families participated in this study (see Table 1 for demographic information). The inclusion criteria were: (a) typically developing (TD) siblings between 12 to 18 years old who had a sibling diagnosed with ASD; (b) willing to share/express their experience dealing with their sibling with ASD; and (c) parents willing to provide consent. The exclusion criteria were (a) TD siblings with reported speech, language, and/or hearing problems; and (b) TD siblings with reported psychosocial or mental health issues. Of these 14 participants, six were first-child, seven were middle-child, and one was the youngest child. For the sake of clarification, different symbols are added to the subjects' identification number to represent the TD siblings who are from the same family. For instance, $\mathrm{S}^{*}, \mathrm{~S}^{*}$ and $\mathrm{S} 6 *$ are siblings of the same brother with ASD. S4! and S7! share the same brother and sister with ASD. S9** and S12** are siblings of the same brother with ASD, and $\mathrm{S} 13++$ and $\mathrm{S} 14++$ are siblings of the same brother with ASD. The participants were recruited through either the university's audiology and speech clinic $(n=7)$, Facebook of the Malaysian Autism Association $(n=3)$, a special education teacher $(n=2)$, or a community-based rehabilitation center $(n=2)$. This study was approved by the university's research ethics board (NN-2020-014) and all parents signed the informed consent form, allowing their children to participate in the study. The purpose of the study was explained clearly to the participants and their parents while they read through the research information sheet. Participants were also well-informed on the voluntary basis of their participation and future use of the data obtained from them. Once the participants gave their verbal consent to participate this study, the parents signed the consent form.

\section{Procedure}

Given the focus on subjective experiences in qualitative inquiry, semi-structured interviews were used to explore the participants' perspectives. Key interview questions were developed based on a review of the literature (Benderix \& Sivberg, 2007; Canha, 2010; Habelrih et al., 2018). These key questions were designed to explore the challenges faced by siblings of individuals with ASD, their 
Table 1 Participants' Demographic Information

\begin{tabular}{llllll}
\hline Subject & Age (years) & Gender & Sibling with ASD & $\begin{array}{l}\text { Age of Sibling } \\
\text { with ASD (Years) }\end{array}$ & $\begin{array}{l}\text { Involvement in } \\
\text { Sibling's SLP } \\
\text { session }\end{array}$ \\
\hline S1 & 13 & M & Elder brother & 21 & No \\
S2 & 14 & F & Younger brother & 8 & No \\
S3 & 13 & M & Younger brother & 7 & No \\
S4 & 13 & F & Younger brother, sister & 8 and 5 & Yes \\
S5 $^{*}$ & 18 & M & Younger brother & 7 & No \\
S6 & 17 & F & Younger sister & 7 & No \\
S7 & 17 & F & Younger brother, sister & 8 and 5 & No \\
S8 & 17 & F & Younger brother & 6 & Yes \\
S9 & 13 & F & Younger sister & 5 & Yes \\
S10 & 15 & F & Younger brother & 15 & No \\
S11 & 15 & F & Younger sister & 13 & Yes \\
S12 & 19 & F & Younger brother & 5 & No \\
S13 & 17 & F & Younger sister & 13 & No \\
S14 & 17 & F & Younger sister & 13 & No \\
\hline
\end{tabular}

S3*, S5* and S6* are siblings of the same brother with ASD, S4! and S7! are siblings of the same brother and sister with ASD, S9** and S12** are siblings of the same brother with ASD, and $\mathrm{S}^{*} 3^{++}$and $\mathrm{S} 14^{++}$are siblings of the same brother with ASD coping strategies, and the effect of these challenges on their relationships (Appendix 1).

The interviews were conducted in Bahasa Malaysia (national language in Malaysia) and English (the second most widely used language in Malaysia) by the second author, who is a fluent bilingual (Bahasa Malaysia/English), fourth-year trained speech-language pathologist (SLP). The interviewer used the strategies of summarizing, reflecting, and clarifying during the interview to ensure shared understanding between the participants and the interviewer. To reduce language barriers during the interview, the language of the interview was not pre-determined. However, considering that all participants were of Malay ethnicity, the interviewer used Bahasa Malaysia to begin the conversation. In the case of code-switching, the interviewer continued with the language that the participants used. The duration of each interview ranged between 30-45 min.

The first five interviews (S1, S2, S3*, S4!, S6*) were conducted face-to-face in the participants' homes. A handphone audio recorder (Vivo Y53) was set on the table to record the conversation. The remainder of the interviews $\left(\mathrm{S} 5 *, \mathrm{~S} 7\right.$ !, $\left.\mathrm{S} 14^{++}\right)$were conducted via telephone due to the COVID-19 movement control restriction order. All interviews were conducted during a time that was most convenient to the participants, given that all of them were bound to an online school schedule during the COVID-19 movement control restriction order. Siblings of the same individual with ASD were interviewed separately to obtain detailed individual responses to the questions.

\section{Data Analysis}

On average, interviews lasted approximately half an hour (mean $=31.94, \mathrm{SD}=9.74$, range $=25-51 \mathrm{~min}$ ). All raw data (i.e., audio recordings, interview transcripts) were named to represent the unique identifier of the participants (e.g., participant code). Raw data were stored in a central repository (a secure network location with folders for each type of raw data) to provide an audit and a means of confirming our data analysis and interpretations of adequacy as a team. Audio recordings of the interviews were transcribed by two undergraduate SLP students (Student A-second author, Student B-a second year student). The accuracy of the transcript was checked using $20 \%$ of all audio and transcripts for consistent information. Student B randomly selected about 10-min of recordings from each interview to transcribe. The transcriptions from Student A and Student B were then printed out and compared by the second author. Transcripts with any discrepancies were coded as " 0 " and consensus was counted as " 1 ". Of these 14 transcriptions, one discrepancy was noticed, resulting in $93 \%$ in agreement.

Audio recordings of the interviews were transcribed and analyzed using Braun and Clarke's (2006) six phases of the thematic analysis model. Criteria for trustworthiness were addressed during each phase of the thematic analysis. The credibility of analysis was further enhanced by having two researchers analyze each data set (Nowell et al., 2017). The first author led the analysis, conducting consensus coding with the second author at each of the six stages of the process. First, the first and second authors read and re-read 
the transcriptions to familiarize themselves with the data, writing down their initial ideas. Second, these two authors completed line-by-line consensus coding from a randomly selected transcript. Ideas and the incidence of recurring topics in the data were identified, and discrete codes were assigned to represent these. The second author then coded the remaining transcripts. Third, the first and second authors reviewed the initial codes, sorting them into potential themes and sub-themes. Fourth, the two authors discussed all the themes and sub-themes, relating them back to the initial codes to show how the patterns of codes developed to be indicative of a recurring or meaningful theme. Fifth, the first, second, and third authors defined and named these themes. Each individual theme was analyzed in detail and discussed during several team meetings until a consensus was reached. This process of peer debriefing helped the team explore their personal insights into the research findings to ensure a thorough analysis of all aspects of the data was achieved. Finally, the third author read and confirmed the selected themes and sub-themes again. All the themes are discussed in the discussion section and serve as the basis for the results reported here.

\section{Results}

Five themes were derived from the interviews with TD siblings of individuals with ASD (Table 2). The first theme, "Communication challenges: understanding and expressing," captured the difficulties faced by TD siblings in their attempts to communicate with their siblings with ASD. The second theme, "I will guide him": strategies for communication breakdown and behaviors, focused on how the siblings responded to communication breakdowns and behavior manifested by their siblings with ASD. The third theme, "I feel sad..." focused on emotional reaction, explored how the TD siblings dealt with their emotions when facing the behavior manifested (e.g., tantrums, shouting, aggressive, etc.) by their siblings with ASD. The fourth theme, "Just keep it to myself, or ignore", highlighted psychological coping strategies used by TD siblings. The final theme, "Sometimes I feel grateful" revealed how the challenges affected the relationship between TD siblings and their siblings with ASD. Quotes are provided in this paper by the TD siblings in colloquial English, which are reported verbatim. However, translations from the Malay language are reported in standard English.

\section{Theme 1: Communication Challenges: Understanding and Expressing}

One of the biggest challenges reported by our respondents was communication difficulties with their siblings with ASD. Eight respondents agreed that the lack of understanding shown by their sibling with ASD limited their on-going conversations, resulting in a communication breakdown. They reported that their siblings had difficulties attending to conversations, understanding instructions, comprehending vocabulary, or maintaining a specific topic. For example, S11 stated that "The limit in communicating with my brother is usually because he struggles to listen to us and to understand what we are trying to say", while S1 reported, "There are words that they understand and did not understand."

Four siblings reported that they sometimes lacked the knowledge of what they could do to communicate better or interact with their siblings. S5* and $\mathrm{S} 9 * *$ stated that they could not understand what their siblings were trying to express to them. S5* said, "Apart from that, we also do not understand what he wants." S9** expressed that, "I do feel frustrated because he tries to tell us something, but we

Table 2 Themes and subthemes from TD siblings with ASD

\begin{tabular}{ll}
\hline Themes & Subthemes \\
\hline Communication challenges: understanding and expressing & - Unable to maintain a conversation \\
& - Lacking knowledge of how to interact \\
"I will guide him": strategies for communication breakdown and behaviors & - Trial and error \\
& - Approaching \\
& - Calm down \\
& - Scolding \\
"I feel sad...": emotional reaction & - Patience and explain \\
& - Negative emotions \\
& - Mixed emotions \\
"Just keep it to myself, or ignore": psychological coping strategies & - Self-regulation \\
& - Self-isolate or change focus \\
& - Getting used to it
\end{tabular}


cannot understand what he is trying to tell us." These challenges led to the inability of the TD siblings to give appropriate responses to their siblings with ASD, as indicated by S10, "I feel like it is hard. I do not know how to teach him" and S4! reported, "I also did not know what to do."

Of the four respondents who had experience attending speech-language therapy sessions with their siblings with ASD, all of them reported that they had a better understanding of their sibling's condition. For example, S10 said, "I learned how to massage him and how to play with him. At first, I did not know, but after my mom explained it to me, and I joined the therapy session (speech therapy), then I understand my brother's condition." Another respondent agreed that it is important to become involved in the therapy session. S6 ${ }^{*}$ reported:

Yes, it's important (to join therapy sessions) because the sibling should also understand what they are going through. Pretty much a lot (to understand sibling with ASD). Most of it. I'll usually just like look at her behavior and I just like (to understand her behavior). I know (I understand why she behaves like that). And sometimes my mom tells me. We learn that we have to cooperate more and help our sibling that has a condition like that $(A S D)$.

\section{Theme 2: "I Will Guide Him": Strategies for Communication Breakdown and Behaviors}

In order to repair communication breakdowns, TD siblings reported using a variety of communication techniques, including forcing, explaining, asking, comforting, using familiar words, and repeating words or instructions. For example, $\mathrm{S}^{*}$ and $\mathrm{S} 12 * *$ reported that they forced their siblings with ASD to complete the task that they intended them to do. S3* explained, "Usually he would not listen to our instructions. He understands us but just would not do it depending on his mood. Usually I will force him (when he refused to do something)". Similar experiences were also reported by S12**: "For example, when he does not want to wear his clothes after a shower, I will usually ask him to do it until he does it, although sometimes he seems to be in his own world". S6* reported that she would explain and tell the sibling to listen to the instructions: "Sometimes, I need to talk to them, tell them and ask them to be quiet or sit quietly." Like S6*, S10 reported, "I will talk properly to him. If he does not listen to me, I will make a disliking face. I will guide him the proper way as I told him not to do wrong things, like beating people."

When there was a breakdown during a conversation, $\mathrm{S}^{*}$, $\mathrm{S} 13^{++}$, and $\mathrm{S}_{14}{ }^{++}$reported they would ask their siblings questions to confirm what their siblings were trying to convey. This let them know whether their sibling understood them or not, as well as what their sibling wanted. For example, $\mathrm{S}_{13}{ }^{++}$said, "I will sit in front of him. I will talk in my language. He may understand, but he did not speak. I will repeat for a few times if he did not understand my question." Another strategy was comforting the sibling. According to S5*: "If he cries badly, I will coax him for a while, then he will pull our hand if he really wants the item." $\mathrm{S} 9$ ** reported using and repeating familiar words that were used by her sibling to find out what he needed, stating: "I will use the words that he may understand, repeat a few times."

Other siblings attempted using other creative ways to find the best strategies that worked for their siblings to communicate with them effectively. For example, S4! reported, "I will show one thing. For example, if usually when I give him bread, he will keep quiet, next time if I give him bread and he does not keep quiet, I will try other things" and S7! stated:

For an example, if I say dog and she doesn't understand it, I will try to express it in as many ways as possible and try to make the sound, because my sister watches YouTube ${ }^{T M}$ so she might know the sound, or anything, or maybe I will draw it out.

When asked how they managed the manifested behaviors, most of the TD siblings reported their own strategies to deal with their siblings with ASD. For example, S10 would remind the sibling with ASD of the "do's and don'ts" and then calm him down afterwards, "Like, scold him. After I scold him, he will come to me, then I will comfort him. Tell him that it is not good to say that (bad words)." S12 $2^{* *}$ reported, "Usually when his sister scold him, he wanted to get back to her. So, I will scold him. Then I will warn him as I afraid that he will act too much." S3 ${ }^{*}$, however, stressed that he would be patient when dealing with his brother with ASD, "Have to be patient. When he freezes his body, need to be patient." S10 stated, "I will remind him like... adik (meaning younger brother), you cannot do this. Then he will understand." S6 ${ }^{*}$ commented that she enjoyed the moment, although sometimes she did get annoyed with the behavior, "Sometimes fun. Sometimes when they don't want to listen, I feel like...I'm getting annoyed."

\section{Theme 3:“I Feel Sad...": Emotional Reaction}

TD siblings revealed a variety of emotional experiences towards the behavior shown by their siblings with ASD, including negative and mixed feelings. Most of them dealt with mixed emotions that included anger, pity, sadness, and guilt. Some siblings (S1, S2, S10, S9**) showed negative emotions towards the inappropriate behaviors manifested by their siblings with ASD. For example, S2 said, "Sometimes I feel mad with his behavior. But I still need to understand his condition." while S5* reported, "Feels like I don't know 
what to do because I don't know why he gets mad. Sometimes, when he refused to potty trained, I will feel angry." Meanwhile, S9** $^{* *}$ stated, "I feel angry because he likes to hit people, pull one's hair."

$\mathrm{S} 14^{++}$admitted that, apart from getting angry, "I do scold him. Because sometimes he doesn't want to listen to us when he acted that way." She continued, "feels like...pity because he is different from us," implying her sibling is different from ordinary people. S10 felt pity toward her brother with ASD when he had done something to hurt himself. The siblings also reported feeling sad when they witnessed the behavior manifested by their siblings. S11 was unhappy when she could not do much to manage her sibling's behavior. She said, "he cries and blames himself, hit his head, so I feel like crying. Sometimes, I feel sad because I cannot control him." While crying, S12** expressed, "Because when I watched him blame himself, I start to...I feel sad because he is unlike his peers." S3* stated, "I feel sad...but eventually, as I handle him, I feel I can deal with this" and S9** said, "At first I felt quite sad, but I tell myself he is not a normal kid. He is a special kid. When I say that, it makes me more motivated to guide him." $\mathrm{S}_{1} 3^{++}$, on the other hand, felt guilty that her brother manifested inappropriate behaviors due to her teasing him:

Sometimes he acted that way also because of me. Sometimes I accidentally get mad at him and I always tease him until he becomes like that (showing undesired behavior). I do feel guilty, but I did not apologize (while giggling).

\section{Theme 4: "Just Keep it to Myself, or Ignore": Psychological Coping Strategies}

When asked how they managed their emotional reactions, the TD siblings reported that they kept their feelings to themselves, as stated by $\mathrm{S}_{13}^{++}$, "like be silent for a few days, and then I'll be okay. I also cannot scold them...so, just keep it to myself." Several of them reported that they self-isolated to manage their emotional reactions. When these siblings decided to isolate themselves, they either cried or ranted silently (S2), "Sit in my room, shut in myself, and cry. I have a box. I will write on a piece of paper, then, I will tear the paper and put it inside the box. So, that way, I feel relieved." Some TD siblings did not want to show their emotions to family members, as reported by $\mathrm{S}_{14}{ }^{++}$, "It occurs (hide feelings). Like... sometimes he acts like that in front of the family. If he doesn't want to listen even after I talk to him, I will ignore him." $\mathrm{S} 5{ }^{*}$, on the other hand, chose to ignore the whole situation, "Usually, I will just sit, and do nothing."

Some respondents stated they did not have any specific way to cope because it did not bother them, or they embraced their sibling's differences. For instance, $\mathrm{S} 9^{* * *}$ said, "Nothing specific because I kind of don't mind." $\mathrm{S} 14^{++}$explained, "Sometimes, we talk to ourselves, like...we need to understand that our brother is different, so don't easily get mad. Need always to remind him, you can do this, you cannot do this." Some of them eventually accepted the nature of ASD and motivated themselves to help their sibling with ASD.

They also distracted themselves by doing other things. S3* said, "Usually I will complete the work first before running away. If I run away first before doing the work, the stress will come later." S4! stated, "I just play games", while S12** reported that "Sometimes, I feel like... keep myself away... do our own things." S7! reported she would reset her mindset, "So I just mainly try to keep myself in a mindset that it is going to be a bit hard with him, so you need to be patient... that is what I always say to myself", which allowed her to be more understanding of her brother's condition.

With some TD siblings (S7!, S9**, S10, $\mathrm{S} 14^{++}$), they got used to the behavior and understood the different learning nature of their siblings with ASD. For example, S7! reported, "I am used with my brother's behavior that was hyper. I will just face it if he was shouting or not. Or if he is too loud, I will ask him to be quiet, he will understand." S9** said, "Eventually is okay, because we know that it is hard for him to learn something. So, I feel normal."

\section{Theme 5: "Sometimes I Feel Grateful”: Impact on Relationship}

When asked about their bond with their siblings with ASD, 9 out of the 14 TD siblings said that it helped forge a closer bond between them and their siblings with ASD. For respondents S2, S3*, and S6*, an ambivalent relationship emerged between them and their sibling with ASD. $\mathrm{S} 2$ said, "It makes us closer when I am away from him. He will feel sad; he will ask about me." S3* reported that, "If throughout the day his mood is okay, then our relationship is okay. Sometimes, when he did something that is not okay and I feel very mad, then we will fight." S6* indicated that, "Sometimes we did fight. But it is fine, I will try to make up with him. Sometimes, I need to give in with my younger siblings."

Several siblings (S7!, S9**, S10, S12**) reported a closer relationship by observing and helping their siblings. For example, S7! revealed, "I don't feel like it distances the bond, but it makes me wanted to help him more, like to calm him down and do not be too hyper. So, that makes me get closer to them." Likewise, S9** said, "Now I will tag along, because I don't have anything to do at home (due to COVID19 lockdown). So, whenever I have the chance to guide for his speech, I will do my best." Meanwhile, $\mathrm{S}_{1}{ }^{++}$taught her brother to improve his expressive skills, contributing to their close relationship. She said, "I think I become closer with him because before this I don't talk much with him and 
he also did his own thing. Now, he can talk to us, can laugh together, so become much closer."

Other TD siblings explained that their close relationships with their siblings with ASD developed after spending more quality time with them, either by looking after them (S4!, S11), preventing unwanted behaviors (S5*), or sharing an interest $\left(\mathrm{S}_{14}{ }^{++}\right)$. For example, S5* expressed, "because I want to prevent him from doing weird stuff, so we spent more time with him." S4! said, "Sometimes I feel grateful to have this kind of sibling because I will become closer to him because I often take care of him," while S11 reported, "We become closer because we spend more time together."

\section{Discussion}

In this study, we explored the communication challenges that can occur as a result of having a sibling with ASD and their impact on relationships in sibling interactions from the perspectives of normally developing siblings of individuals with ASD. Overall, the participants shared commonalities in the challenges they faced when communicating with their siblings with ASD, their emotional reactions, their psychological coping strategies toward the communication challenges, the ways they overcome communication breakdowns, and the impact of these challenges on their relationships with their siblings with ASD.

As delineated in Family Systems Theory, resilience was apparent in our findings, considering that our respondents made efforts to spend time with their ASD siblings. This could also be due to the COVID-19 lockdown, which provided opportunities for siblings to interact more and spend additional time together. Furthermore, it also reflects the active efforts our respondents made to spend time with each other, maintain normal routines, and use practical methods to overcome challenges (Seligman \& Darling, 2007). Another positive facet indicated in Family Systems Theory, traumatic growth, is applicable to our families with a child with ASD. Traumatic growth is a process, beginning with the realization, upon receiving the diagnosis of ASD, that these children will not develop typically, to experiences over time of relief, validation, and acceptance of having a member with ASD (Samios et al., 2012). Our TD siblings appeared to experience traumatic growth, based on their reported emotions of sadness, guilt, and anger with their siblings with ASD. Stressful experiences may impact on the sense of closeness and empathy within the family, supporting the notion within Family Systems Theory that all members in the family interact to influence each other. Hence, viewing the family as a whole system rather than focusing only one single family member is important when assessing and providing rehabilitation services. Outcomes from this study could inform allied healthcare professionals, considering that they provide knowledge about the disorder, as well as information and emotional support, and help families respond to grief (Pinkerton \&Dolan, 2007; Wetherby \&Woods, 2006; Woods \& Brown, 2011). Specifically, more support for families from therapists is needed, including informing them of the disorder, teaching them techniques to overcome communication challenges, and involving TD siblings in the interventions. Future research on the interrelations of various aspects of Family Systems Theory (e.g. individual, subsystemic and macroscopic) should also be targeted to understand how these subsystems affect family functioning.

\section{Training TD Siblings on Strategies for Better Communication Interactions}

Communication challenges are prominent issues for individuals with ASD as they encounter difficulties with both expressive and receptive language, as well as language use in social contexts (Macha et al., 2020). As expected, findings in the present study revealed that difficulties with understanding often led to communication breakdowns. Our respondents reported that "forcing" and "scolding" were sometimes communication reactions toward their ASD siblings' unfavorable behaviors. Considering that a direct, emotional response such as "scolding" is not a typical way of interaction among siblings in Malaysian culture, this suggests that these TD siblings encountered frustrations with the challenges of handling their personal developmental growing up as an adolescent, while facing difficulties communicating with their ASD siblings. In addition, the TD siblings reported a lack of knowledge regarding strategies on how to communicate in order to better understand the needs of their sibling with ASD, which negatively affected their interactions. Similar findings have been reported, in which TD siblings often complain that they do not know how to play and interact with their siblings with ASD (Ferraioli et al., 2012). Our results raised two points that may be important for an intervention designed to support TD adolescent siblings. First, in order to manage undesired behaviors, providing explanations and controlling one's emotional reactions (e.g., anger) may be most beneficial. Second, professionals and parents need to acknowledge the importance of TD siblings' roles in their brother's or sister's future, since the TD siblings could possibly continue to provide support for them when their parents are no longer able to do so.

Supporting and educating TD siblings on strategies to engage with their brother or sister with ASD is necessary for better interaction skills (Moyson \& Royers, 2012; Kryzak, $\&$ Jones, 2017). Such training could help encourage communication and engagement with siblings with ASD during play situations (Jones et al., 2019). Based on case study research, which has shown that siblings are usually involved 
in taking care of family members with special needs (Gan, 2017), the communication capabilities of the TD siblings in this study are particularly important for the creation of rewarding, caring relationships with their siblings with ASD and enhancing the well-being of both.

\section{Inclusion of TD Siblings in Rehabilitation Interventions}

Providing dynamic support and promoting positive sibling relationships is vital for both children with ASD and their siblings, and such relationships should develop when they are young. Given that the closeness of sibling relationships earlier in life is associated with supporting adults with disabilities later in life (Avieli et al., 2019), intervention that includes young TD siblings may help the TD siblings learn to view their brother's or sister's perspectives. TD siblings who had more knowledge about ASD and a stronger support network practiced better coping strategies and reported a more positive experience of their relationship with their sibling with ASD (Jones et al., 2019). Four out of the 14 siblings (28.6\%) in this study who were previously involved in the speech-language treatment of their siblings with ASD also reported a better understanding of their siblings' condition and limitations in communication as they learned effective techniques directly from the speech-language therapist during treatment sessions. Considering that siblings spend more time with each other, it is possible for the professional to support their interaction and further foster generalization of speech and language skills (Wright, 2019). Previous studies have found that exposing TD siblings to the activities and interventions in which their sibling with autism participates can help them better understand how their sibling with ASD is functioning, and aids the TD siblings in the learning of more effective strategies to cope with communication breakdowns (Ferraioli et al., 2012; McConkey et al., 2020; McCullough \& Simon, 2011; Sheikh et al., 2019). Through understanding, facilitating, and developing empathy, intervention could potentially build a stronger sibling relationship and a better outcome for individuals with ASD and their TD siblings.

\section{Factors Influencing Emotional Experience}

One of the main problems that the TD siblings experience is the behavioral issues of their siblings with ASD, such that negative behaviors can decrease their desire to spend much time with their siblings (Yavuz \& Şafak, 2019). The siblings in the current study, however, experienced a variety of emotions towards the behaviors manifested by their siblings with ASD, including anger, sadness, guilty, pity, and neutral feelings. Similar findings have been previously found, where TD siblings reported mixed emotional experiences, including tension and frustration while also feeling a sense of empathy (Pavlopoulou, \& Dimitriou, 2019; Petalas, 2012). Mixed findings were also reported in another study, where some children were found to be positively affected (e.g., high sense of self-concept) by having a sibling with ASD while others reported negative experiences (e.g., loneliness; low levels of prosocial behavior) (Habelrih et al., 2018). These results are likely due to diagnostic factors such as the severity of ASD, the amount of problematic behaviors by the sibling with ASD, and the family support given to the TD sibling (Meadan et al., 2010; Tomeny et al., 2017). Moreover, the age range may also obscure age-related changes in sibling relationships (Orsmond et al., 2009). According to Family Systems Theory, a stable emotional network forms among TD and ASD siblings with the inclusion of other family members, especially parents. This emotional network serves a function in their family system and is transmitted through multi-generations. Therefore, interventions for various issues in ASD will be more effective with the understanding of the TD siblings, their family systems as a whole, and trans-generationally (e.g., how communication interaction patterns and emotional responses towards challenging and traumatic experiences within the family have been formed and passed on from one generation to another), rather than merely focusing on the individuals with ASD. The rehabilitation intervention will be more holistic and inclusive with various family systems' perspectives and approaches.

\section{Strategies to Cope and Manage Challenging Behaviors}

In terms of coping with the challenging behaviors manifested by their siblings with ASD, the TD siblings in this study often used avoidant coping strategies by isolating themselves in order to let out their emotions and distract themselves with other things. This present finding is similar to a previous study, where distraction served as an effective coping strategy (Johnson et al., 2020; Ross \& Cuskelly, 2006). Taken together, this suggests that TD siblings of children with ASD are aware of the levels of distress and anger that ASD aggressive behaviors produce in them and choose to cope with these situations by actively controlling their emotional reactions. In addition, they either recognize that the aggressive actions are due to ASD, and/or they escape momentarily from the impact of the situation.

Despite the negative feelings experienced, these TD siblings acknowledged the difficult nature of their siblings' challenging behaviors and utilized a variety of strategies to manage the behavior, including focusing on preventing bad experiences, encouraging good behavior, or teaching their sibling new skills (Gorjy et al., 2017). According to Tsai et al. (2018), these methods of adapting are related to more 
elevated levels of coping difficulties. Although these types of coping strategies might lead to worrisome long-term effects on the siblings, they are acceptable as defensive reactions to situations that are not entirely understood by the TD siblings or those situations which they are unable to manage (Donaldson et al., 2000).

The TD siblings in this study internalized their feelings and self-isolated themselves or chose to focus on other tasks when coping with challenging behaviors. This also occurred when the TD siblings did not want to show their frustration in front of family members in order to avoid worrying their parents. Parents can play a significant role in providing important information to their TD children regarding their siblings with ASD in order to help ward against negative feelings. As a result, TD siblings can become more empathetic and understanding towards their siblings and others with ASD (Tsai et al., 2018).

\section{Quality Time with Siblings due to COVID-19}

The TD siblings in this study believed that they nurtured a close, but ambivalent relationship with their siblings with ASD. These were reflected in the quality time they spent with their siblings with ASD, such as looking after them, doing fun activities together, and managing their behavior. In fact, one of the respondents commented that due to the COVID-19 movement control restrictions, he was able to spend more time playing with his sister, resulting in a closer relationship. In addition, they often had the urge to help their siblings with ASD develop skills like their typically developing peers. These positive qualities highlight the fact that the TD siblings were still able to identify and maintain what could be considered typical characteristics in their sibling relationships despite the challenges they encountered as a result of the behavioral and emotional difficulties associated with ASD.

\section{Culture and Belief on Sibling Relationship}

Although their experience impacted some aspects of their lives negatively, the TD siblings chose to cherish special episodes of their lifelong learning, including increased awareness, compassion, tolerance, and understanding of disabilities (Tsai et al., 2018). Nevertheless, this was often a perspective that evolved over time and was related to an increase in quality of the sibling relationship (Gorjy et al., 2017). It is worth mentioning that all respondents in this current study were of Malay ethnicity. For Malays, the child is seen as a form of a test from God (Jegatheesan \& Fowler, 2010). In the present study, our respondents viewed their siblings with ASD as a divinely-given, and therefore important challenge which motivated them to value their siblings' condition and be patient when dealing with their sibling. As a result, and despite the occasional frustrations noted earlier, cultural and religious beliefs may play a part in the acceptance of a child's condition (Chu et al., , 2020a, 2020b). Malaysia is a multicultural-multilingual country. Future research should determine whether cultural or religious beliefs affect one's perceptions on growing old with siblings with ASD. Asian culture also places importance on the view of "face", which is the concern that having a child with disabilities will bring shame and disgrace to the family (Sue \& Zane, 1987). Understanding how the family structure and support impacts siblings' interactions may need further study.

\section{Limitations and Future Studies}

The perspectives on the challenges and barriers faced by adolescent TD siblings of children with ASD in this investigation are specific to the small sample of participants who were involved in this study and do not necessarily represent the views of all adolescents in other parts of Malaysia, much less in other countries. However, in-depth information was provided by all the participants, who were from various backgrounds and experiences. To the authors' knowledge, these are the first preliminary interviews conducted in Malaysia with TD adolescents with siblings with ASD.

The communication challenges and their impact on sibling relationships were obtained from the perspectives of siblings with and without experience in the participation of speech-language therapy sessions with their siblings with ASD. However, the former only consists of four out of the 14 participants. Thus, in-depth conclusions on the impact of the TD siblings' involvement in speech-language therapy sessions on the development of the siblings' relationships, or improvement in function cannot be reported in this study. Future studies that focus on the impact of siblings' involvement in rehabilitation interventions (e.g., speech-language, occupational, physical therapies) with their siblings with ASD are suggested in order to shed light for professionals considering the involvement of siblings in therapy in the management of children with ASD. In the present study, information regarding the severity of ASD, expressive language ability, and other comorbidities was not requested from parents. As a result, it is difficult to determine whether the mixed emotional responses from the respondents in this study were due to these factors in their siblings and/or the degree of problematic behaviors. Although differences in emotional reactions between the male and female siblings were observed, no in-depth conclusions on the impact of gender towards emotional reactions could be made in the current study. Considering that this study was limited by an imbalance in our sample, which consisted of mostly females (11 females to 3 males), 
it was not possible to further examine the gender effect on emotional reactions, even though differences between the genders has been previously reported (Guidotti et al., 2021). In addition, further research is needed to investigate the experiences of TD siblings older or younger than a sibling with ASD given that there are limited studies on these two different groups.

Due to the COVID-19 pandemic lockdown, the use of telephone interviewing was an economical, time-, and money-saving way to obtain data (Cachia, \& Millward, 2011). Although the interviewer reported the lack of facial expressions and non-verbal cues in the telephone interviews, she did not notice any differences between the responses provided over the phone and the face-to-face interviews. The adolescents interviewed via telephone gave as much information as the adolescents that were interviewed face-to-face, sharing their personal experiences with the interviewer. There is the possibility that the adolescents interviewed over the telephone may not have had as much privacy as the face-to-face respondents. When our interviewer interviewed the participants at home, they understood that their answers were to be completely confidential, and parents could choose to either leave the room or close the door if they felt the child's answers might be overheard. In the case of the adolescents interviewed over the phone, although we explained to their parents that we preferred for the adolescents to speak with us privately, we could not control whether the parents, who might be interested in what their children shared, were attending the interview besides the participant. Future studies that examine responses between face-to-face and phone interviews are warranted.

\section{Conclusion}

The current study indicated that TD adolescents struggled to communicate with their siblings with ASD. The contributing factors were due to a lack of knowledge about communication strategies that could be used with individuals with ASD. This led to a "trial and error" approach on the part of the TD siblings when a communication breakdown occurred, and the experience of various emotional reactions when coping with their siblings' behaviors. Future studies should focus on the well-being of TD adolescents when dealing with their lifelong relationships with their siblings with ASD. Findings from this study can be used to inform the practice of speechlanguage pathology and other allied health professionals, as well as future research. Exploring the perceptions and experiences of TD siblings of children with ASD could enhance support services for not only the children with ASD, but their TD siblings as well.

\section{Appendix 1: Interview Questions}

\section{Part A: Demographic}

1. How old are you?

2. How many brothers/sisters do you have?

3. How old are they?

4. How old is your brother/sister who has ASD? What is her/his name?

5. Can you describe your autistic brother/sister's behavior for me?

6. How do you deal with it (5)? Please describe.

\section{Part B: Challenges, Coping Strategies and Impacts on Interaction}

1. How does your sibling's behavior affect your relationship with him/her?

2. How do you usually communicate with (name)?

3. What do you go through when communicating/interacting with (name)?

4. What other challenges do you face when communicating with him/her?

5. How do you overcome the challenges?

6. How does that make you feel about your brother/sister? Describe.

7. When you worry about (...), what do you do?

8. Is there anything else that you want to share with me?

Acknowledgments This study was supported in part by the University's incentive grant (GP-2020-K021244, first author). The authors thank all the respondents who participated in this study.

Author contributions SYC, CHG, and VF contributed to the design and the computational framework on this study. SNZK carried out the interviews. SYC, SNZK, VF, and CHG contributed to the analysis of the results and to the writing of the manuscript. DH provided critical feedback and helped shape the research, analysis and manuscript. All authors discussed the results and contributed to the final manuscript.

\section{Declarations}

Conflict of interest The authors declare no conflicts of interest.

Research Involving Human Participants This research was approved by the university approved by the university's research ethics board (NN-2020-014).

Informed Consent All parents signed the informed consent form, allowing their children to participate in the study. 


\section{References}

Avieli, H., Band-Winterstein, T., \& Araten Bergman, T. (2019). Sibling relationships over the life course: Growing up with a disability. Qualitative Health Research, 29(12), 1739-1750.

Benderix, Y., \& Sivberg, B. (2007). Siblings' experiences of having a brother or sister with autism and mental retardation: A case study of 14 siblings from five families. Journal of Pediatric Nursing, 22(5), 410-418.

Braun, V., \& Clarke, V. (2006). Using thematic analysis in psychology. Qualitative Research in Psychology, 3(2), 77-101.

Cachia, M., \& Millward, L. (2011). The telephone medium and semistructured interviews: A complementary fit. Qualitative Research in Organizations and Management: An International Journal, 6(3), 265-277.

Canha, M. (2010). Siblings of children with Autism: An exploratory study of sibling concerns and coping strategies. Undergraduate Review, 6(1), 42-46.

Chiarotti, F., \& Venerosi, A. (2020). Epidemiology of autism spectrum disorders: a review of worldwide prevalence estimates since 2014. Brain Sciences, 10(5), 274.

Chu, S. Y., Mohd Normal, S. N. S. A. B., McConnell, G. E., Tan, J. S., \& Joginder Singh, S. K. D. (2020a). Challenges faced by parents of children with autism spectrum disorder in Malaysia. Speech, Language and Hearing, 23(4), 221-231. https://doi.org/10.1080/ 2050571X.2018.1548678

Chu, S. Y., Park, H., Lee, J., Shaharuddin, K. K. B., \& Gan, C. H. (2020b). Self-stigma and its associations with stress and quality of life among Malaysian parents of children with autism. Child: Care, Health, \& Development, 46(4), 485-494. https://doi.org/ 10.1111/cch. 12771

Cicirelli, V. G. (1994). Sibling relationships in cross-cultural perspective. Journal of Marriage and the Family, 56, 7-20.

Cridland, E. K., Jones, S. C., Stoyles, G., Caputi, P., \& Magee, C. A. (2016). Families living with autism spectrum disorder: Roles and responsibilities of adolescent sisters. Focus on Autism and Other Developmental Disabilities, 31(3), 196-207.

Donaldson, D., Prinstein, M. J., Danovsky, M., \& Spirito, A. (2000). Patterns of children's coping with life stress: Implications for clinicians. American Journal of Orthopsychiatry, 70(3), 351-359.

Dongola, E. O. (2018). Living with a sibling diagnosed with autism spectrum disorder: an interpretative phenomenological analysis (Doctoral dissertation).

Ferraioli, S. J., Hansford, A., \& Harris, S. L. (2012). Benefits of including siblings in the treatment of autism spectrum disorders. Cognitive and Behavioral Practice, 19(3), 413-422.

Gan, C. H. (2017). Clinical psychology support for siblings of the people with special needs - Reviewing the concept of 'young carer' with case studies. Bulletin of Center for Clinical Psychology and Human Development, Kyushu University., 9, 217-228.

Gordon-Pershey, M., \& Hodge, A. M. (2017). Communicative behaviours of sibling dyads composed of a child with autism and a typically developing child. Journal of Interactional Research in Communication Disorders, 8(2), 246-273.

Gorjy, R. S., Fielding, A., \& Falkmer, M. (2017). "It's better than it used to be": Perspectives of adolescent siblings of children with an autism spectrum condition. Child \& Family Social Work, 22(4), $1488-1496$.

Guidotti, L., Musetti, A., Barbieri, G. L., Ballocchi, I., \& Corsano, P. (2021). Conflicting and harmonious sibling relationships of children and adolescent siblings of children with autism spectrum disorder. Child: Care, Health and Development, 47(2), 163-173.

Habelrih, E. A., Hicks, R. E., \& Vanstone, D. M. (2018). A study of the wellbeing of siblings of children with Autism Spectrum Disorders: Sibling efficacy, positive and negative affect, and coping strategies. International Journal of Psychological Studies, 10(2), $102-113$

Hall, S. A., \& Rossetti, Z. (2018). The roles of adult siblings in the lives of people with severe intellectual and developmental disabilities. Journal of Applied Research in Intellectual Disabilities, 31(3), 423-434.

Henderson-Rudling, J. M. (2019). In the Shadows of Autism: A Collective Case Study on Life with a Sibling with Autism in a Military Family. Doctoral Dissertations and Projects. 2154. https://digit al-commons.liberty.edu/doctoral/2154

Jegatheesan, B., Miller, P. J., \& Fowler, S. A. (2010). Autism from a religious perspective: A study of parental beliefs in South Asian Muslim immigrant families. Focus on Autism and Other Developmental Disabilities, 25(2), 98-109.

Johnson, M. T., Williamson, R. L., Casey, L. B., Stockton, M., \& Elswick, S. (2020). Sibling relationships when one sibling has ASD: A preliminary investigation to inform the field and strengthen the bond. Children and Youth Services Review, 116, 105190.

Jones, E. A., Fiani, T., Stewart, J. L., Sheikh, R., Neil, N., \& Fienup, D. M.(2019). When one sibling has autism: Adjustment and sibling relationship. Journal of Child and Family Studies, 28(5), $1272-1282$.

Kale, M., \& Aslan, D. (2020). The caregiving practices of nomadic Yuruk Turkmen families for their children in terms of developmental well-being: an ecocultural perspective. Early Child Development and Care. https://doi.org/10.1080/03004430.2020.17181 23

Koukouriki, E., \& Soulis, S. G. (2020). Self-reported Health-related quality of life (HRQOL) and anxiety among greek school-age siblings of individuals with autism spectrum disorders (ASD) in relation to parental mental health and social support. Journal of Autism and Developmental Disorders, 50, 2913-2930.

Kryzak, L. A., \& Jones, E. A. (2017). Sibling self-management: Programming for generalization to improve interactions between typically developing siblings and children with autism spectrum disorders. Developmental Neurorehabilitation, 20(8), 525-537.

Laghi, F., Lonigro, A., Pallini, S., Bechini, A., Gradilone, A., Marziano, G., \& Baiocco, R. (2017). Sibling relationships and family functioning in siblings of early adolescents, adolescents and young adults with autism spectrum disorder. Journal of Child and Family Studies, 27(3), 793-801.

Leedham, A. T., Thompson, A. R., \& Freeth, M. (2020). A thematic synthesis of siblings' lived experiences of autism: Distress, responsibilities, compassion and connection. Research in Developmental Disabilities, 97, 103547.

Lord, C., Brugha, T. S., Charman, T., Cusack, J., Dumas, G., Frazier, T., \& Taylor, J. L. (2020). Autism spectrum disorder. Nature Reviews Disease Primers, 6(1), 1-23.

Macha, V., Simui, F., \& Muzata, K. K. (2020). Parents and Teachers Experiences of Managing Peculiar Psychosocial Behaviours of Learners with Autism Spectrum Disorder in Selected Special Units in Lusaka, Zambia.

McConkey, R., Cassin, M. T., \& McNaughton, R. (2020). Promoting the socialinclusion of children with asd: a family-centred intervention. Brain Sciences, 10(5), 318.

McCullough, K., \& Simon, S. R. (2011). Feeling heard: A support group for siblings of children with developmental disabilities. Social Work with Groups, 34(3-4), 320-329.

McHale, S. M., Updegraff, K. A., \& Feinberg, M. E. (2016). Siblings of youth with autism spectrum disorders: Theoretical perspectives on sibling relationships and individual adjustment. Journal of Autism and Developmental Disorders, 46(2), 589-602.

Meadan, H., Stoner, J. B., \& Angell, M. E. (2010). Review of literature related to the social, emotional, and behavioral adjustment of siblings of individuals with autism spectrum disorder. Journal of 
Developmental and Physical Disabilities, 22, 83-100. https://doi. org/10.1155/2012/949586

Moss, P., Eirinaki, V., Savage, S., \& Howlin, P. (2019). Growing older with autism-The experiences of adult siblings of individuals with autism. Research in Autism Spectrum Disorders, 63, 42-51. https://doi.org/10.1016/j.rasd.2018.10.005

Moyson, T., \& Roeyers, H. (2012). 'The overall quality of my life as a sibling is all right, but of course, it could always be better' Quality of life of siblings of children with intellectual disability: the siblings' perspectives. Journal of Intellectual Disability Research, 56(1), 87-101.

Nuckolls, C. W. (1993). An introduction to the cross-cultural study of sibling relations. In C. W. Nuckolls (Ed.), Siblings in South Asia: Brothers and sisters in cultural context (pp. 19-44). Guilford Press.

Noonan, H., O’Donoghue, I., \& Wilson, C. (2018). Engaging with and navigating limbo: Lived experiences of siblings of adults with autism spectrum disorders. Journal of Applied Research in Intellectual Disabilities, 31(6), 1144-1153.

Nowell, L. S., Norris, J. M., White, D. E., \& Moules, N. J. (2017). Thematic analysis: Striving to meet the trustworthiness criteria. International Journal of Qualitative Methods, 16(1), 1-13.

Orsmond, G. I., Kuo, H. Y., \& Seltzer, M. M. (2009). Siblings of individuals with an autism spectrum disorder: Sibling relationships and wellbeing in adolescence and adulthood. Autism, 13, 59-80.

Pavlopoulou, G., \& Dimitriou, D. (2019). 'I don't live with autism; I live with my sister'. Sisters' accounts on growing up with their preverbal autistic siblings. Research in Developmental Disabilities, 88, 1-15.

Pavlopoulou, G., \& Dimitriou, D. (2020). In their own words, in their own photos: Adolescent females' siblinghood experiences, needs and perspectives growing up with a preverbal autistic brother or sister. Research in Developmental Disabilities, 97, 103556.

Petalas, M. A., Hastings, R. P., Nash, S., Dowey, A., \& Reilly, D. (2009). "I like that he always shows who he is": The perceptions and experiences of siblings with a brother with autism spectrum disorder. International Journal of Disability, Development and Education, 56(4), 381-399.

Petalas, M. A., Hastings, R. P., Nash, S., Reilly, D., \& Dowey, A. (2012). The perceptions and experiences of adolescent siblings who have a brother with autism spectrum disorder. Journal of Intellectual and Developmental Disability, 37(4), 303-314.

Pinkerton, J., \& Dolan, P. (2007). Family support, social capital, resilience and adolescent coping. Child \& Family Social Work, 12(3), 219-228.

Ross, P., \& Cuskelly, M. (2006). Adjustment, sibling problems and coping strategies of brothers and sisters of children with autistic spectrum disorder. Journal of Intellectual and Developmental Disability, 31(2), 77-86.

Samios, C., Pakenham, K. I., \& Sofronoff, K. (2012). Sense making and benefit finding in couples with Asperger syndrome: An application of the actor-partner interdependence model. Autism, 16(3), 275-292.

Seligman, M., \& Darling, R. B. (2007). Ordinary Families, Special Children: A Systems Approach to Childhood Disability. Guilford Press.
Shali, A. A., \& Patil, M. (2017). Affect of child factors on the sibling relationship of normal child with mentally challenged sibling. International Journal of Home Science, 3, 242-246.

Sheikh, R., Patino, V., Cengher, M., Fiani, T., \& Jones, E. A. (2019). Augmenting sibling support with parent-sibling training in families of children with Autism. Developmental Neurorehabilitation, 22(8), 542-552.

Stocker, C. M., \& McHale, S. M. (1992). The nature and Family Correlates of Preadolescents' Perceptions of their Sibling Relationships. Journal of Social and Personal Relationships, 9(2), 179-195.

Sue, S., \& Zane, N. (1987). The role of culture and cultural techniques in psychotherapy: A critique and reformulation. American Psychologist, 42, 37-45.

Sumari, M., Baharudin, D. F., Khalid, N. M., Ibrahim, N. H., \& Ahmed Tharbe, I. H. (2019). Family functioning in a collectivist culture of Malaysia: A qualitative study. The Family Journal, 28(4), 396-402.

Tomeny, T. S., Barry, T. D., Fair, E. C., \& Riley, R. (2017). Parentification of adultsiblings of individuals with autism spectrum disorder. Journal of Child and Family Studies, 26(4), 1056-1067.

Travers, H. E., Carlton, M. E., \& Carter, E. W. (2020). Social connections among siblings with and without intellectual disability or autism. Intellectual and Developmental Disabilities, 58(1), 19-33.

Tsai, H. W. J., Cebula, K., Liang, S. H., \& Fletcher-Watson, S. (2018). Siblings' experiences of growing up with children with autism in Taiwan and the United Kingdom. Research in Developmental Disabilities, 83, 206-216.

Ward, B. (2016). Sibling experiences: Living with young persons with autism spectrum disorders. Pediatric Nursing, 42(2), 69-76.

Willig, C., \& Stainton-Rogers, W. (2008). The SAGE handbook of qualitative research in psychology. SAGE Publications Ltd. https://doi. org/10.4135/9781848607927

Wright, B. M., \& Benigno, J. P. (2019). Autism spectrum disorder and sibling relationships: Exploring implications for intervention using a family systems framework. American Journal of SpeechLanguage Pathology, 28(2), 759-767.

Woods, J. J., \& Brown, J. A. (2011). Integrating family capacity building and child outcomes to support social communication development in young children with autism spectrum disorder. Topics in Language Disorders, 31(3), 235-246.

Wu, K., Kim, J. H., Nagata, D. K., \& Kim, S. I. (2018). Perception of sibling relationships and birth order among Asian American and European American emerging adults. Journal of Family Issues, 39(13), 3641-3663.

Yavuz, M., \& Şafak, P. (2019). Investigating the time children with autistic spectrum disorders spend with their typically developing siblings. Universal Journal of Educational Research, 7(8), 1795-1807.

Publisher's Note Springer Nature remains neutral with regard to jurisdictional claims in published maps and institutional affiliations. 